\title{
Assessment of White Matter Injury and Outcome in Severe Brain Trauma
}

\section{A Prospective Multicenter Cohort}

\author{
Damien Galanaud, M.D., Ph.D., ${ }^{\star}$ Vincent Perlbarg, Ph.D., † Rajiv Gupta, M.D., Ph.D., \\ Robert D. Stevens, M.D., Ph.D.,§ Paola Sanchez, M.D.,\|l Eléonore Tollard, M.D.,\# \\ Nicolas Menjot de Champfleur, M.D., ${ }^{\star \star}$ Julien Dinkel, M.D., † Sébastien Faivre, M.D. $\dagger \dagger$ \\ Gustavo Soto-Ares, M.D., \$t Benoit Veber, M.D. Ph.D., §§ Vincent Cottenceau, M.D., III \\ Françoise Masson, M.D.,\#\# Thomas Tourdias, M.D., Ill Edith André, M.D., ${ }^{* \star}$

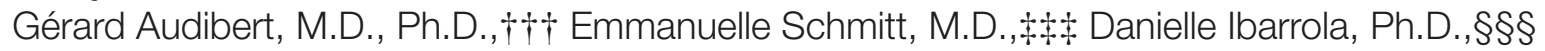 \\ Frédéric Dailler, M.D., IIIII Audrey Vanhaudenhuyse, Ph.D.,\#\#\# Luaba Tshibanda, M.D., ${ }^{\star \star \star \star \star ~}$ \\ Jean-François Payen, M.D., Ph.D., $\dagger \dagger \dagger \dagger$ Jean-François Le Bas, M.D., Ph.D., $+\$+\neq$

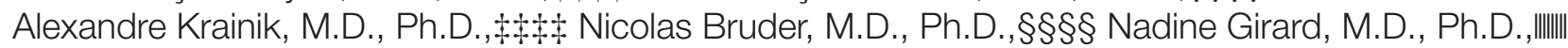 \\ Steven Laureys, M.D., Ph.D.,\#\#\# Habib Benali, Ph.D., \$ Louis Puybasset, M.D., Ph.D.\#\#\#\#; for the \\ Neuro Imaging for Coma Emergence and Recovery Consortium ${ }^{\star \star \star \star \star}$
}

\begin{abstract}
Background: Existing methods to predict recovery after severe traumatic brain injury lack accuracy. The aim of this study is to determine the prognostic value of quantitative diffusion tensor imaging (DTI).
\end{abstract}

* Associate Professor, Department of Neuroradiology, Pitié Salpêtrière Hospital, \& Université Pierre et Marie Curie (Paris VI), Boulevard de l'Hôpital, Paris, France. † Research Associate, INSERM, UMRS, Université Pierre et Marie Curie-Paris, Paris, France. $\ddagger$ Doctor, Department of Neuroradiology, Massachusetts General Hospital, Boston, Massachusetts. § Associate Professor, Division of Neuroscience Critical Care, Johns Hopkins University School of Medicine, Baltimore, Maryland. II Doctor, Neurosurgical Intensive Care Unit, Pitié Salpêtrière Hospital, Assistance Publique-Hôpitaux de Paris, Paris, France. \# Doctor, Department of Neuroradiology, Centre Hospitalier Universitaire, Rouen, France. ** Doctor, Department of Neuroradiology, Guy de Chauliac Hospital, Montpellier, France. $\dagger \dagger$ Doctor, Department of Anesthesia and Surgical Intensive Care Unit, $+\neq$ Doctor, Department of Neuroradiology, Roger Salengro Hospital, Lille, France. §§ Professor, Surgical Intensive Care Unit, Centre Hospitalier Universitaire, Rouen, France. IIII Doctor, Department of Neuroradiology, \#\# Doctor, Department of Anesthesia and Surgical Intensive Care Unit, Centre Hospitalier Universitaire, Bordeaux, France. **** Doctor, Department of Anesthesia \& Intensive Care Unit, Guy de Chauliac Hospital, Montpellier, France. $\dagger \dagger \dagger$ Professor, Department of Anesthesia and Surgical Intensive Care Unit, $\$+$ Doctor, Department of Neuroradiology, Centre Hospitalier Universitaire, Nancy, France. \$s Research Associate, CERMEP, Pierre Wertheimer Neurological \& Neurosurgical Hospital, Bron, France. IIIII Surgical Intensive Care Unit, Pierre Wertheimer Neurological \& Neurosurgical Hospital, Lyon, France. \#\# Research Associate, Cyclotron Research Center, ***k* Doctor, Department of Neuroradiology, University of Liège, Liège, Belgium. $+\dagger+$ Professor, Department of Anesthesia and Surgical Intensive Care Unit, $\$+\$+$ Professor, Department of Neuroradiology, Michallon Hospital, La Tronche, France. sss Professor, Department of Anesthesia \& Intensive Care Unit, IIIIII Professor, Department of Neuroradiology, La Timone Hospital, Marseille, France. \#\#\# Professor, Neurosurgical Intensive Care Unit, Pitié Salpêtrière Hospital, Assistance Publique-Hôpitaux de Paris, Copyright $\odot$ 2012, the American Society of Anesthesiologists, Inc. Lippincott Williams \& Wilkins. Anesthesiology 2012; 117:00-00

\section{What We Already Know about This Topic}

- Traumatic brain injury is a major public health problem, and current methods to predict long-term outcome and resource utilization are not strong

- Measuring white matter injury using magnetic resonance diffusion tensor imaging might improve prediction but has not been studied in a multicenter fashion

\section{What This Article Tells Us That Is New}

- In a multicenter study of 105 patients with traumatic brain injury, diffusion tensor imaging, using a normalization process across different machine types, increased the accuracy of long-term outcome prediction compared with standard clinical and imaging approaches

and Université Pierre et Marie Curie-Paris, Paris, France ****ok See appendix for a supplementary list of investigators.

Received from Assistance Publique-Hôpitaux de Paris, Paris, France; Université Pierre et Marie Curie-Paris 6, Paris, France; INSERM, Paris, France; CNRS, Paris, France; Massachusetts General Hospital, Boston, Massachusetts; Harvard University, Boston, Massachusetts; Johns Hopkins Hospital, Baltimore, Maryland; Johns Hopkins University, Baltimore, Maryland; and Université de Liège, Liège, Belgium. Submitted for publication June 27, 2012. Accepted for publication September 17, 2012. This work was funded by a grant from the French ministry of health (ProjetHospitalier de Recherche Clinique registration \#P051061 (2005)), Paris, France, and from the "Agence Nationale de la Recherche," Paris France, for the program "investissements d'avenir" under agreement $n^{\circ}$ ANR-10-IAIHU-06 for the Paris Institute of Translational Neurosciences-IHU-A-ICM. Other support was provided from institutional and/or departmental sources.

Address correspondence to Dr. Galanaud: Department of Neuroradiology, Pitié Salpêtrière Hospital, 47 Boulevard de l'Hôpital, 75013 Paris, France. galanaud@gmail.com. This article may be accessed for personal use at no charge through the Journal Web site, www.anesthesiology.org.

$\diamond$ This article is featured in "This Month in Anesthesiology." Please see this issue of Anesthesiology, page 9A. 
Methods: In a multicenter study, the authors prospectively enrolled 105 patients who remained comatose at least 7 days after traumatic brain injury. Patients underwent brain magnetic resonance imaging, including DTI in 20 preselected white matter tracts. Patients were evaluated at $1 \mathrm{yr}$ with a modified Glasgow Outcome Scale. A composite DTI score was constructed for outcome prognostication on this training database and then validated on an independent database $(\mathrm{n}=38)$. DTI score was compared with the International Mission for Prognosis and Analysis of Clinical Trials Score.

Results: Using the DTI score for prediction of unfavorable outcome on the training database, the area under the receiver operating characteristic curve was 0.84 (95\% CI: $0.75-0.91$ ). The DTI score had a sensitivity of $64 \%$ and a specificity of $95 \%$ for the prediction of unfavorable outcome. On the validation-independent database, the area under the receiver operating characteristic curve was 0.80 (95\% CI: 0.54-0.94). On the training database, reclassification methods showed significant improvement of classification accuracy $(P<0.05)$ compared with the International Mission for Prognosis and Analysis of Clinical Trials score. Similar results were observed on the validation database.

Conclusions: White matter assessment with quantitative DTI increases the accuracy of long-term outcome prediction compared with the available clinical/radiographic prognostic score.

$S$ EVERE traumatic brain injury (TBI) represents a major public health burden, generally requiring resuscitation in an intensive care unit (ICU) and prolonged rehabilitation. For patients with TBI, there is considerable uncertainty regarding long-term outcome in terms of a broad range of cognitive, behavioral, and functional impairments. Available methods for the prediction of long-term outcome are inaccurate and unreliable, in part because clinical and electrophysiological evaluations are limited by coma or sedation. As a result, decisions regarding therapeutic intensity and goals of care are commonly made on the basis of limited evidence, leading to a potential mismatch between outcomes and resources mobilized to care for a patient, with associated psychological and financial burdens on patients, their families, and society. ${ }^{1}$

The most extensively validated scoring system for TBI outcome is the International Mission for Prognosis and Analysis of Clinical Trials (IMPACT) score. ${ }^{2}$ It is based on a multivariate model that combines clinical, biochemical, and computed tomography (CT) variables at admission to provide a probabilistic estimate of the outcome at 6 months. The IMPACT score is accurate in predicting outcomes in populations of patients with moderate and severe TBI but has limited utility in making decisions regarding any individual patient. It has been proposed that clinical decision-making must be based on additional information that reflects the biological heterogeneity of TBI. ${ }^{3}$

White matter damage, a key feature of TBI, can be identified and quantified with a magnetic resonance imaging (MRI) sequence called diffusion tensor imaging (DTI).
Single-center studies have demonstrated the diagnostic and prognostic value of DTI in patients with TBI. ${ }^{4-6}$ However, for these results to be widely applicable, quantitative MRI methods must account for hardware and software disparities within and across institutions. The goal of the current study was to develop and validate, as a first step, an algorithm based on DTI for outcome prediction in severe TBI in a multicenter setup after implementation of a normalization process. We hypothesized that DTI would significantly increase our predictive ability to discriminate between favorable and unfavorable (death, vegetative state, or minimally conscious state) outcomes at $1 \mathrm{yr}$ compared with the IMPACT score.

\section{Material and Methods}

\section{Study Oversight}

The institutional review boards of participating institutions approved the study. Written informed consent was obtained for all study participants (patient's next of kin during the acute stage, and patients themselves after recovery of consciousness). The protocol was registered on December 2007 (NCT00577954).

\section{Study Design}

We enrolled patients in a prospective observational multicenter cohort between October 2006 and March 2010. Imaging and clinical data from comatose patients with TBI was collected at predetermined time points using agreed upon shared data elements. An outcome prediction model was developed using the information available in the acute setting. We tested the hypothesis that this model can differentiate between patients with favorable and unfavorable outcome with greater accuracy than the IMPACT model alone.

\section{Patients}

Patients were enrolled in ICUs at 10 participating institutions. Inclusion criteria were (1) adult patient between 18 and $75 \mathrm{yr}$ of age; and (2) inability to follow simple commands that could not be explained by sedation at least 7 days, and not more than 45 days, after TBI. Exclusion criteria were (1) moribund patients (expected survival < 24h); (2) physiological instability (e.g., due to hemodynamic instability, increased intracranial pressure, and/or rapidly deteriorating respiratory function) that would preclude MRI scanning; (3) contraindication to the MRI; (4) penetrating head injury; and (5) a central nervous system condition such as stroke, brain tumor, or a neurodegenerative disease preceding TBI. Five to 10 healthy volunteers were recruited at each center to serve as control subjects to account for potential variations in DTI values across centers. ${ }^{7}$

\section{Clinical Data Collection}

Using standardized case report forms, data were collected and stored in a central, web-based, encrypted database. These included patient characteristics, initial clinical status, and cranial CT scan; adverse events associated with MRI scanning; 1-yr outcome using the Glasgow Outcome Scale 
(GOS), the Disability Rating Scale, the extended Glasgow Outcome Score (GOSE), and the modified Rankin Scale. A central study monitor verified all data for accuracy, consistency, and completeness.

\section{Head CT Scan}

Head CT scans were performed within $48 \mathrm{~h}$ after ICU admission and rated using the Marshall score. ${ }^{8}$ When more than one CT scan was obtained, the scan showing the worst radiologic findings was selected and scored.

\section{Brain MRI}

An MRI scan was acquired as soon as a patient met inclusion criteria, and the CT scan was clinically feasible. During MRI acquisition, patient sedation, if any, was continued. At the 10 different sites, MRI scans were performed using 12 scanners with either 1.5 or 3.0 Tesla field strengths and from three manufacturers: GE Healthcare (Milwaukee, WI), Siemens Medical Solutions (Erlangen, Germany), and Philips Medical Systems (Eindhoven, The Netherlands). The precise parameters of each sequence were adapted to the individual scanner type, field strength, coil used, and departmental protocol. The following morphologic sequences were acquired: sagittal localizer, axial T2/FLAIR (Fluid Attenuated Inversion Recovery), axial T2, and T2*, 3D inversion recovery T1. In addition, DTI was acquired in an axial plane perpendicular to the main field B0. The DTI parameters used were field of view of $300 \mathrm{~mm}$, matrix size $96 \times 96$, and slice thickness $3 \mathrm{~mm}$ (resulting in nearly isotropic voxels). Gradient (B1) was applied in at least 12 directions (range 12-50) with a value of $1000 \mathrm{mT} / \mathrm{m}$. A series without the diffusion gradient (the B-zero image) was also acquired.

MRI results, including the morphologic sequences and unprocessed DTI, were assembled in a centrally administered imaging core laboratory at the Pitié-Salpêtrière Hospital (Paris, France). The clinical teams treating the patients had access to all MRI results, with the exception of the DTI data.

\section{MRI Analysis}

All MRI scans were reviewed to check for motion and other artifacts. DTI images were preprocessed using the FSL software. ${ }^{9}$ †十ナ† The diffusion tensor was estimated, and the local diffusion parameters, namely fractional anisotropy (FA), mean diffusivity (MD), axial diffusivity (L1), and radial diffusivity (Lt), were calculated for the entire brain in each patient and control. These parameters were computed from the three estimated eigenvalues that quantify the parameters of water diffusion in three orthogonal directions. ${ }^{10}$ Correction for distortions caused by Eddy currents was performed using the B-zero images.

To make diffusion measures comparable between individuals, the FA, MD, L1, and Lt maps were registered on a $1 \times$ $1 \times 1 \mathrm{~mm}^{3}$ standard space image (MNI152 space) using the

†ナナ†† http://www.fmrib.ox.ac.uk/fsl/. Accessed November 2, 2012
Tract-based Spatial Statistics procedure. ${ }^{11}$ The whole brain was registered using a nonlinear technique, and individual FA, MD, L1, and Lt values were projected on an alignmentinvariant template for the brain. This procedure maps all available information to a common brain template and avoids misalignment between subjects.

The regions-of-interest (ROIs) for DTI analysis were selected from the atlas designed by Mori et al., ${ }^{12}$ the so-called ICBM-DTI-81 white matter atlas. This atlas, which is included in FSL, consists of 48 white matter tracts. For the purpose of this analysis, these were merged in 20 larger regions shown in figure 1 and were used to extract the diffusion parameters. For each patient and control, the average values of FA, MD, L1, and Lt in these 20 ROIs resulted in 80 DTI biomarkers for each subject.

The regional DTI parameter extraction consisted of three steps: a nonlinear registration of FA map to a template (provided by FSL), a projection of FA onto the FA template skeleton representing the centers of all tracts (also provided by FSL), and averaging of FA measures within the 20 ROIs restricted to the skeleton. The resulting maps were checked

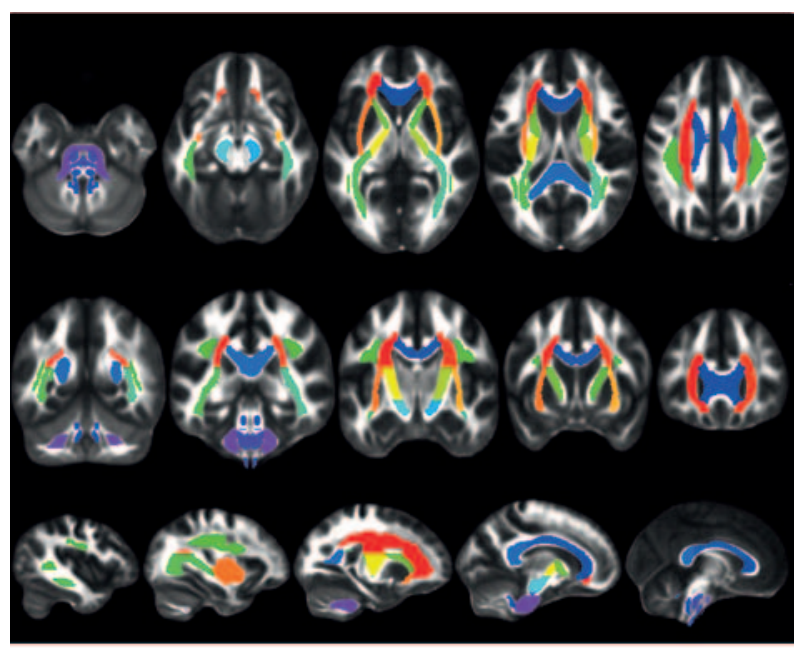

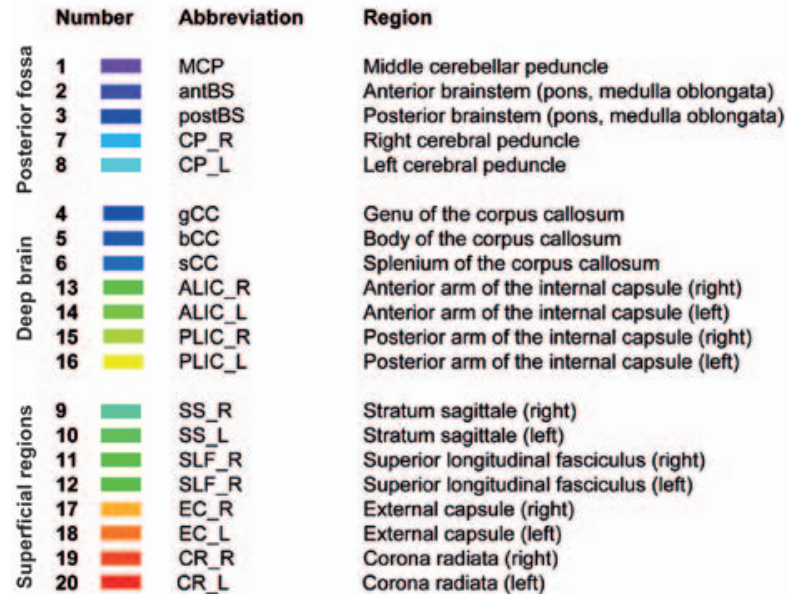

Fig. 1. Automatically segmented white matter tracks for measurement of diffusion tensor imaging variables. 
by Dr. Galanaud; any patient with major distortion was excluded.

\section{DTI Parameters Normalization}

Ninety-nine normal controls underwent the same imaging protocol as that used for the patients. All were free of previous neurological diseases and gave written informed consent to participate in the study. Given the variability in raw DTI values, which arises because of differences in MRI vendors, scanners, and field strengths across centers, a normalization procedure had to be performed. The raw value of each derived diffusion parameter was standardized using the data from control subjects for each center as described below.

The raw DTI parameter values for each patient were normalized with respect to the average of each parameter measured in the control group for that center. Specifically, for each patient, the raw FA, MD, L1, and Lt values in each of the 20 preselected ROIs were divided by the corresponding mean value for the control group from his or her center.

\section{Outcome Measures}

Outcomes were determined via a telephone interview conducted by the investigative team at each participating center. The principal outcome was modified GOS 1 yr after injury. For this research, we modified the GOS (as previously described) ${ }^{6}$ by dividing the score of 3 , which is a heterogeneous category of severe motor and cognitive disabilities, into 3- and 3+ subcategories. The 3- subcategory denotes the minimally conscious state ( as defined by Giacino et al. ${ }^{13}$ ); the $3+$ score represents severe disability excluding minimally conscious state. A score of 1, 2, or 3- at 1 yr was classified as unfavorable, whereas higher scores $(3+, 4$, and 5$)$ were classified as favorable.

Three investigators (Drs. Galanaud, Puybasset, and Sanchez) performed an audit of recorded clinical outcomes to check for data completeness, accuracy, and consistency.

\section{Outcome Prediction Algorithm}

DTI variables (specifically, the radial, axial and MD, and FA) from the 20 preselected regions were integrated with the following eight IMPACT score variables: age, motor score, pupillary reactivity, hypoxia, hypotension, CT classification, subarachnoid hemorrhage, and mass effect from epidural hematoma. Each patient was associated with a class label (favorable or unfavorable outcome), and the above 88 DTI and clinical variables.

Support Vector Machine (SVM), a supervised learning method, was used for classification. ${ }^{14}$ This classification method is known to maintain its reliability when the number of features is close to the number of subjects. SVM classification, implemented via the libsvm library, ${ }^{14}$ 执执 is based on two main concepts: decision planes and the

择扭 http://www.csie.ntu.edu.tw/ cjlin/libsvm/. Accessed November 2, 2012.

$\S \S \S \S$ http://www.r-project.org/. Accessed November 2, 2012. nonlinear mapping. Given a set of input vectors, where each vector is also associated with one of two class labels (e.g., favorable and unfavorable), the goal of classification algorithm is to find the optimal surface that maximizes the margin between the two classes. When this surface is a plane, we get a linear classifier. However, the surface that separates the two classes may not always be linear. To overcome this limitation, SVM transforms the input vectors into a high-dimensional space using a kernel function in such a way that the two classes are separable by a linear hyperplane. The algorithm fits the maximummargin hyperplane in the transformed feature space. The goal of the SVM algorithm is then to optimize the parameters of the kernel function to enable the search for such a maximum-margin hyperplane.

Because of the heterogeneity of the GOS 3 patients, the SVM training process was computed on the multicentric cohort without the GOS 3 patients ( $\mathrm{n}=73$ patients). The relevant variables and the optimal SVM kernel parameters were selected by a joint stepwise and grid search procedure using cross-validation (leave-one-out) maximizing the classification accuracy. In addition to the predicting class label, the classification process also assigned to each patient an estimated probability that he or she belongs to the class of favorable or unfavorable outcome. This probability was termed the DTI score. The IMPACT score was also computed for each patient and compared with the DTI score.

\section{Independent Validation}

Thirty-eight patients and 15 controls described in a previous study $^{6}$ were used as a validation dataset. MRI examinations were performed using the same DTI acquisition parameters but on a different MRI unit. They were blindly processed by an external observer (Dr. Dinkel) using the DTI classification method. The classification model selected with the training database (see previous paragraph) was used to evaluate the DTI score of the patients of this validation dataset. IMPACT scores were also calculated for all patients of this dataset.

\section{Statistical Analysis}

Date are expressed as mean + SD or median (scores). Comparison of two proportions was performed using the chisquare test, comparison of two means was performed using the Student $t$ test, and comparison of several means was performed using one-way multivariate analysis of variance.

The ability of the IMPACT and DTI scores to discriminate between favorable and unfavorable outcomes at $1 \mathrm{yr}$ was evaluated and compared by the area under the receiver operating characteristic (ROC) curve analysis. ${ }^{15}$ The sensitivity of the classifier was calculated for $95 \%$ specificity for unfavorable outcome prediction. In addition, we computed net reclassification improvement and integrated discrimination improvement indices to compare our DTI score with IMPACT ${ }^{16}$ by using R Software. $\S \S \S \S$ Confidence interval 
(95\% CI) of each indice (area under the ROC curve, net reclassification improvement, and integrated discrimination improvement) was provided by bootstraping the studied populations. This provided a large sample of each index, and thus the median and its associated $95 \% \mathrm{CI}$.

All comparisons were two tailed, and a $P$ value of less than 0.05 was considered significant.

\section{Results}

\section{Study Population and Enrolment Pattern}

Of 167 patients enrolled, 33 were excluded from analysis because of suboptimal MRI acquisitions; 19 could not be processed because of a lack of acquisitions of healthy volunteers in two centers. An additional 10 patients were lost to follow-up at $1 \mathrm{yr}$ (fig. 2). Clinical and imaging characteristics of patients are summarized in tables 1 and 2 . Patients were predominantly young adult males with severe TBI at admission. MRI was performed on average $21 \pm 9$ days after trauma (20 \pm 9 days for patients with favorable outcome and $23 \pm 11$ days for patients with unfavorable outcome, $P=0.08$ ). The automatic segmentation software accurately recognized the $20 \mathrm{ROI}$ in all 105 patients, even in the presence of intracranial or subdural hematoma, midline shift or decompressive craniotomy. No adverse events related to MRI scanning were reported.

During the 12-month follow-up period, 21 patients (20\%) died, 14 in the ICU and 7 after ICU discharge. At 1 yr, 40 patients (38\%) had an unfavorable GOS; of these, 21 were dead, 5 were in a vegetative state, and 14 were minimally conscious.

\section{Normalization Process}

For the 99 controls, DTI measures were extracted in each of the 20 preselected regions. The mean DTI measures for the controls were significantly different $(P<0.001)$ between centers before the normalization procedure (data not shown).

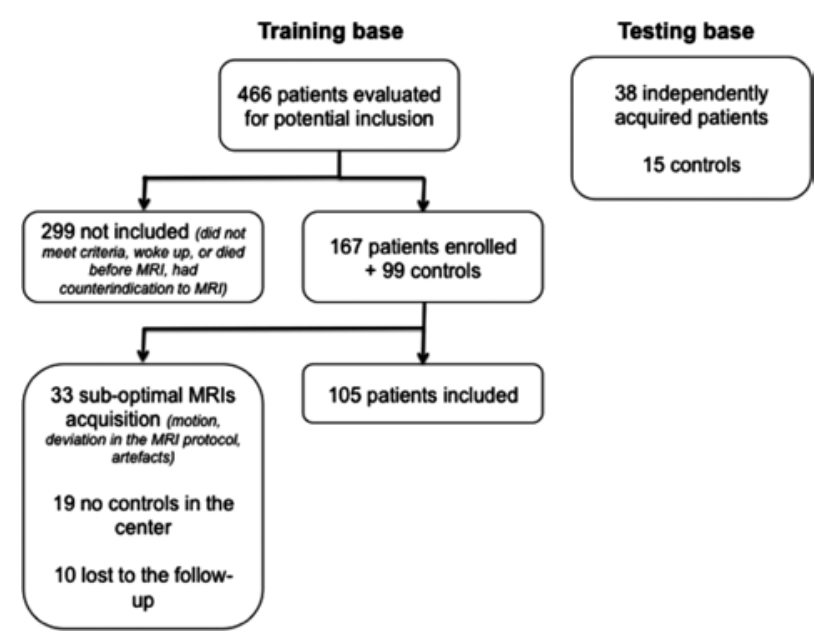

Fig. 2. Flowchart of patient and controls enrolment and MRI. $\mathrm{MRI}=$ magnetic resonance imaging.
This variability in raw DTI values was due to differences in MRI vendors, scanners, and field strengths.

\section{Unfavorable Outcome Prediction}

The ROC curves for the prediction of unfavorable GOS are shown in figure 3 . The best model for prediction of unfavorable outcome used only 32 of the 88 parameters available (80 DTI and 8 clinical) that are summarized in table 3 . As can be seen, all parameters used by this model were indeed DTI metrics.

For the cohort of patients used for deriving the prediction model (training base), area under the ROC curve increased from 0.64 to 0.84 (table 4 ) when the DTI score was used instead of the IMPACT score $(P<0.001)$. The sensitivity for predicting unfavorable outcome, with a specificity of $95 \%$, was $64 \%$. Net reclassification improvement and integrated discrimination improvement indicated also a significant improvement with the DTI score compared with the IMPACT score $(P<0.001)$.

The ROC curve was also plotted using the above-derived prediction model on the independently acquired database of 38 patients with severe TBI (testing base). The area under this ROC curve was 0.80 , and the overall shape of the curve was similar to the one obtained from the training database. Significant improvements from IMPACT to DTI score were also observed for net reclassification improvement $(P=0.04)$ and integrated discrimination improvement $(P=0.009)$. Figure 4 shows the likelihood of unfavorable outcome as a function of the DTI score in the training database. The sigmoidal shape of the DTI score is unmistakable and suggests that low and high scores, an outcome can be assigned with high specificity.

The DTI scores of the patients who survived with unfavorable outcome was not significantly different from the scores of those who died in the ICU $(P=0.75)$. This indicates a lack of systematic selection bias introduced in the ICU by the availability of morphologic MRI.

Figure 5 shows morphologic MRI scans, DTI images, and individual FA values for the 20 regions in two patients, one with favorable outcome and the other with unfavorable outcome. The morphologic images for both patients show widespread signal abnormalities secondary to TBI. The FA values for the patient with favorable outcome are nearly normal, with the exception of subcortical regions 9, 10, and 18 . On the other hand, the patient with unfavorable outcome has markedly decreased FA values (i.e., in the lowest quartile of patients with unfavorable outcome) in all 20 regions. The DTI score for the first patient was 0.17 compared with 0.92 for the patient with unfavorable outcome.

\section{Discussion}

The task of predicting long-term outcome in severe TBI is challenging. Patients with similar clinical and radiologic characteristics in the acute phase may have markedly different outcomes ranging from death to complete recovery, 
Table 1. Patient and Injury Characteristics

\begin{tabular}{|c|c|c|c|}
\hline & $\begin{array}{c}\text { Favorable } \\
\text { Outcome }(n=65)\end{array}$ & $\begin{array}{c}\text { Unfavorable } \\
\text { Outcome }(n=40)\end{array}$ & $P$ Value \\
\hline Age, yr & $36 \pm 16$ & $38 \pm 16$ & $\begin{array}{l}0.20 \\
0.84\end{array}$ \\
\hline $\begin{array}{l}\% \text { Male } \\
\% \text { Dead }\end{array}$ & $\begin{array}{c}53(82 \%) \\
0\end{array}$ & $\begin{array}{l}32(80 \%) \\
21(52 \%)\end{array}$ & $\begin{array}{c}0.84 \\
<0.001\end{array}$ \\
\hline $\begin{array}{l}\text { Associated extracranial injury } \\
\text { Associa }\end{array}$ & $47(72 \%)$ & $31(78 \%)$ & 0.55 \\
\hline \multicolumn{4}{|l|}{ Type of accident (distribution) } \\
\hline - MVA - motorcycle or car & $44(68 \%)$ & $20(50 \%)$ & 0.07 \\
\hline - MVA - pedestrian & $4(6 \%)$ & $6(15 \%)$ & 0.13 \\
\hline - Assault & $3(5 \%)$ & $0(0 \%)$ & 0.16 \\
\hline - Fall & $11(17 \%)$ & $9(23 \%)$ & 0.47 \\
\hline - Other & $3(5 \%)$ & $5(13 \%)$ & 1.13 \\
\hline \multicolumn{4}{|l|}{ Characteristics at admission } \\
\hline - GCS & $6(4-8)$ & $4(3-7)$ & 0.09 \\
\hline - Hypotension & $4(7 \%)$ & $2(5 \%)$ & 0.80 \\
\hline - Hypoxemia & $4(7 \%)$ & $14(36 \%)$ & $<0.0001$ \\
\hline - Mydriasis* & $13(20 \%)$ & $13(33 \%)$ & 0.14 \\
\hline - $\mathrm{Hb}, \mathrm{g} / \mathrm{dl}$ & $12.6 \pm 2.4$ & $12.5 \pm 2.0$ & 0.47 \\
\hline - ASAT, U/I & $120 \pm 152$ & $95 \pm 64$ & 0.18 \\
\hline - ALAT, U/I & $100 \pm 144$ & $67 \pm 54$ & 0.11 \\
\hline - Lactates, mM & $2.4 \pm 1.4$ & $2.2 \pm 1.0$ & 0.21 \\
\hline - Chronic alcohol abuse, \% & $6(9 \%)$ & $6(15 \%)$ & 0.36 \\
\hline \multicolumn{4}{|l|}{ Marshall Score $(\dagger)$} \\
\hline - Diffuse injury I-II & $27(42 \%)$ & $18(45 \%)$ & 0.72 \\
\hline - Diffuse injury III & $9(14 \%)$ & $5(13 \%)$ & 0.84 \\
\hline - Diffuse injury IV & $3(5 \%)$ & $1(3 \%)$ & 0.58 \\
\hline - Mass lesion (operated or not) & $16(25 \%)$ & $15(38 \%)$ & 0.15 \\
\hline \multicolumn{4}{|l|}{ Hematoma } \\
\hline - Epidural & $11(22 \%)$ & $6(15 \%)$ & 0.79 \\
\hline - Subdural & $26(40 \%)$ & $14(35 \%)$ & 0.60 \\
\hline - Subarachnoid hemorrhage & $48(74 \%)$ & $34(85 \%)$ & 0.17 \\
\hline \multicolumn{4}{|l|}{ Brain edema } \\
\hline \multicolumn{4}{|l|}{ - Midline shift } \\
\hline - Absence & $45(66 \%)$ & $26(65 \%)$ & 0.65 \\
\hline 。 $0-5, \mathrm{~mm}$ & $11(17 \%)$ & $5(13 \%)$ & 0.54 \\
\hline • $5-10, \mathrm{~mm}$ & $6(9 \%)$ & $1(3 \%)$ & 0.17 \\
\hline$\circ>10 \mathrm{~mm}$ & $4(6 \%)$ & $8(20 \%)$ & 0.03 \\
\hline Compressed third ventricle & $21(33 \%)$ & $19(48 \%)$ & 0.11 \\
\hline Compressed basal cisterns & $20(31 \%)$ & $21(53 \%)$ & 0.02 \\
\hline Contusion & $49(75 \%)$ & $27(67 \%)$ & 0.38 \\
\hline Neurosurgical intervention & $17(26 \%)$ & $14(35 \%)$ & 0.33 \\
\hline
\end{tabular}

Data are expressed as mean + SD or median or number (25-75 interquantile range).

*At least one side. $(\dagger)$ worst score from any CT performed in the first $48 \mathrm{~h}$ after admission.

ALAT = alanine aminotransferase; ASAT = aspartate aminotransferase; CT = computed tomography; GCS = Glasgow Coma Scale;

MVA = motor vehicle accident.

with intermediate states of impaired consciousness or neuropsychological dysfunction. Neurological examination is hindered in the acute setting by factors such as endotracheal intubation, sedation, and systemic metabolic alterations. Such examinations, therefore, lack accuracy and reliability in measuring the severity of TBI and in predicting outcome. The prognostic value of cranial CT scan is also limited. ${ }^{16}$
The IMPACT score, based on a multivariate model combining clinical, biochemical, and CT scan characteristics, has been developed from a dataset of more than 9,000 patients and has been validated externally against similar large patient populations. ${ }^{17}$ However, this score does not have the discriminative power needed for clinical decision-making in individual patients. ${ }^{3}$ 
Table 2. Distribution of Patients across Various Outcome Categories on Four Measurement Scales

\begin{tabular}{|c|c|c|}
\hline & $\begin{array}{c}\text { Favorable } \\
\text { Outcome } \\
(n=65)\end{array}$ & $\begin{array}{c}\text { Unfavorable } \\
\text { Outcome } \\
(n=40)\end{array}$ \\
\hline Death in ICU & NA & $14(35 \%)$ \\
\hline Death after ICU discharge & NA & $7(18 \%)$ \\
\hline \multicolumn{3}{|l|}{ GOS } \\
\hline - I (death) & NA & $21(52 \%)$ \\
\hline - II (vegetative state) & NA & $5(12 \%)$ \\
\hline - III- (MCS) & NA & $14(35 \%)$ \\
\hline - III+ (severe disability) & $16(25 \%)$ & 0 \\
\hline $\begin{array}{l}\text { - IV (moderate } \\
\text { disability) }\end{array}$ & $22(34 \%)$ & 0 \\
\hline - V (no sequella) & $27(42 \%)$ & 0 \\
\hline \multicolumn{3}{|l|}{ GOS extended (GOSE) } \\
\hline - 1 (dead) & 0 & $21(52 \%)$ \\
\hline - 2 (vegetative state) & 0 & $5(12 \%)$ \\
\hline $\begin{array}{c}\text { - } 3 \text { (lower severe } \\
\text { disability) }\end{array}$ & $9(14 \%)$ & $9(35 \%)$ \\
\hline $\begin{array}{l}\text { - } 4 \text { (upper severe } \\
\text { disability) }\end{array}$ & $9(14 \%)$ & $5(12 \%)$ \\
\hline $\begin{array}{l}\text { - } 5 \text { (lower moderate } \\
\text { disability) }\end{array}$ & $5(8 \%)$ & 0 \\
\hline $\begin{array}{l}\text { - } 6 \text { (upper moderate } \\
\text { disability) }\end{array}$ & $17(26 \%)$ & 0 \\
\hline - 7 (lower good recovery) & $13(20 \%)$ & 0 \\
\hline - 8 (upper good recovery) & $12(18 \%)$ & 0 \\
\hline \multicolumn{3}{|l|}{ Modified rankin scale } \\
\hline - VI (death) & 0 & $21(52 \%)$ \\
\hline - V (severe disability) & $5(8 \%)$ & $17(42 \%)$ \\
\hline $\begin{array}{l}\text { - IV (moderately severe } \\
\text { disease) }\end{array}$ & $11(17 \%)$ & $3(8 \%)$ \\
\hline - III (moderate disability) & $12(18 \%)$ & 0 \\
\hline - II (slight disability) & $18(28 \%)$ & 0 \\
\hline $\begin{array}{l}\text { - I (no significant } \\
\text { disability) }\end{array}$ & $13(20 \%)$ & 0 \\
\hline - 0 (no symptoms) & $6(9 \%)$ & 0 \\
\hline \multicolumn{3}{|l|}{ Disability rating scale } \\
\hline - Mean & 4 & 19 \\
\hline - Median & 3 (1 to 6$)$ & 20 (16 to 24$)$ \\
\hline
\end{tabular}

Data are median (25-75 interquartile range) or number (percentages). GOS = Glasgow Outcome Scale; GOSE = extended Glasgow Outcome Score; $\mathrm{ICU}=$ intensive care unit; $\mathrm{MCS}$ = minimally conscious state; NA = not applicable.

A number of studies have described the utility of conventional MRI sequences, ${ }^{18-20}$ diffusion-weighted imaging, ${ }^{21,22}$ DTI, ${ }^{23,24}$ and susceptibility-weighted imaging ${ }^{25,26}$ in patients with TBI. This literature demonstrates the superiority of MRI over CT for the visualization of lesions. ${ }^{18}$ It is also known that damage to critical areas such as the brainstem ${ }^{5,27}$ or corpus callosum ${ }^{24}$ is indicative of poor prognosis. However, current imaging methods do not allow one to reliably
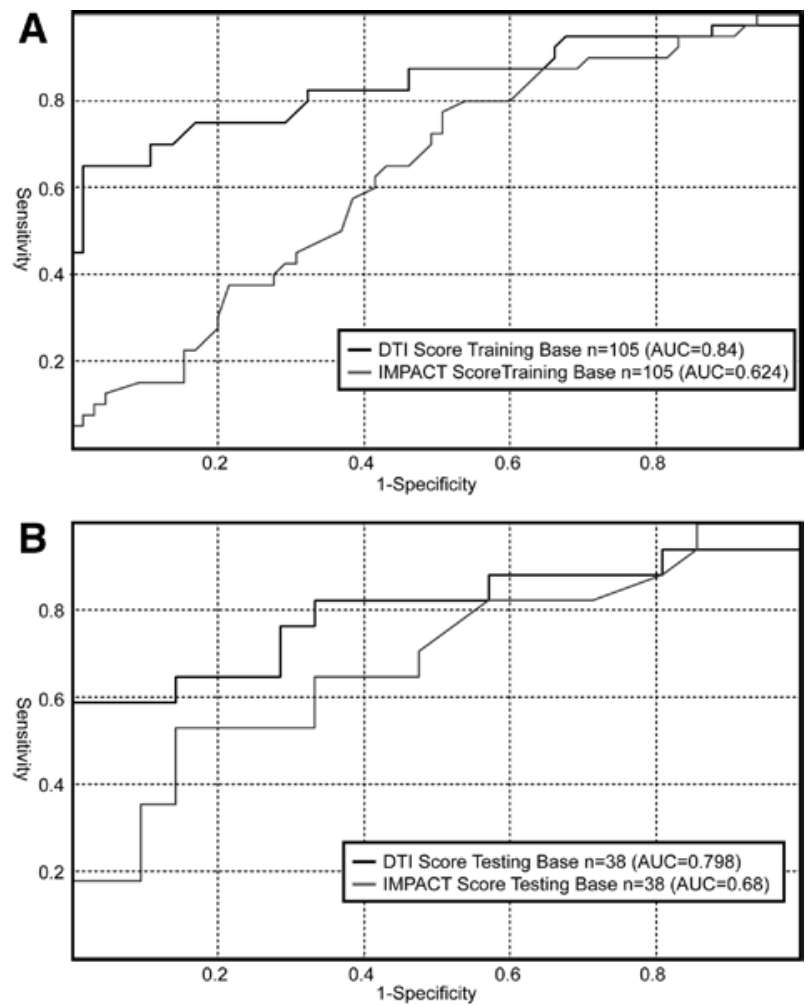

Fig. 3. Receiver operating characteristics of IMPACT and DTI score for predicting coma outcome on the modified Glasgow Outcome Scale in the training $(A)$ and testing $(B)$ databases. $A U C=$ area under the curve; $D T I=$ diffusion tensor imaging; IMPACT = International Mission for Prognosis and Analysis of Clinical Trials.

predict the long-term clinical outcome of an individual comatose patient.

There is extensive evidence that diffuse axonal injury is a hallmark of severe TBI. Recent experimental data indicate that white matter abnormalities detected using DTI correlate closely with neuropathological evidence of diffuse axonal injury. ${ }^{28,29}$ In a prospective study, Sidaros et al. evaluated 30 patients with severe TBI at 8 weeks and 12 months after injury. They found that decreased regional FA at 8 weeks was predictive of unfavorable outcome at 12 months. DTI evidence of white matter damage is more marked in subjects with moderate and severe TBI compared with those with mild TBI, with longlasting changes detectable years after injury. ${ }^{30,31}$

Our prospective multicenter cohort is the largest study so far to demonstrate that the extent and severity of white matter damage evaluated in the acute setting is a major predictor of outcome after severe TBI. Based on this observation, we have developed a prognostic model that integrates quantitative diffusion variables into a composite DTI score for predicting outcome. This score, which is based solely on DTI variables, has a better prognostic accuracy than the IMPACT score that uses clinical and CT variables.

For prediction of unfavorable outcome, the area under the ROC curve was 0.64 for the IMPACT score; this metric 
Table 3. Summary of the 32 Diffusion Tensor ImagingDerived Metrics Used to Build the DTI Score, Classified by Order of Importance

\begin{tabular}{lc}
\hline \multicolumn{1}{c}{$\begin{array}{c}\text { Name of the ROI Ordered per } \\
\text { Weight in the Statistical Model }\end{array}$} & $\begin{array}{c}\text { No. Voxels } \\
\text { per ROI }\end{array}$ \\
\hline 1. FA cerebral peduncle $R$ & 22 \\
2. FA cerebral peduncle $L$ & 23 \\
3. FA splenium of corpus callosum & 85 \\
4. FA posterior limb of internal capsule $L$ & 32 \\
5. FA genu of corpus callosum & 65 \\
6. FA anterior limb of internal capsule $L$ & 29 \\
7. FA posterior brainstem & 47 \\
8. Lt splenium of corpus callosum & 85 \\
9. FA external capsule $L$ & 53 \\
10. FA sagittal stratum $L$ & 49 \\
11. FA posterior limb of internal capsule $R$ & 31 \\
12. FA body of corpus callosum & 116 \\
13. Lt cerebral peduncle $L$ & 23 \\
14. Lt posterior limb of internal capsule $L$ & 32 \\
15. Lt cerebral peduncle $R$ & 22 \\
16. Lt genu of corpus callosum & 65 \\
17. Lt corona radiata $L$ & 135 \\
18. FA anterior limb of internal capsule $R$ & 29 \\
19. Lt external capsule $L$ & 53 \\
20. L1 body of corpus callosum & 116 \\
21. Lt posterior limb of internal capsule $R$ & 31 \\
22. Lt corona radiata $R$ & 138 \\
23. FA anterior brainstem & 46 \\
24. L1 posterior brainstem & 47 \\
25. FA sagittal stratum $R$ & 22 \\
26. Lt sagittal stratum $L$ & 23 \\
27. Lt anterior brainstem & 46 \\
28. Lt posterior brainstem & 47 \\
29. FA middle cerebellar peduncle & 77 \\
30. L1 posterior limb of internal capsule $L$ & 32 \\
31. L1 sagittal stratum $L$ & 23 \\
32. L1 corona radiata $L$ & 138 \\
\hline & \\
\hline &
\end{tabular}

The right column gives the mean size (in Voxels) of the ROI from which they are derived.

$\mathrm{DTI}=$ diffusion tensor imaging; $\mathrm{FA}$ = fractional anisotropy;

$\mathrm{L}=$ left; $\mathrm{L} 1=$ axial relaxivity; $\mathrm{Lt}=$ transverse relaxivity;

$\mathrm{R}=$ right; $\mathrm{ROI}=$ regions-of-interest.

increased to 0.84 when DTI score was used for prognostication. A high specificity, potentially at the expense of a lower sensitivity, is critical for the prediction of unfavorable outcome. The ROC analyses presented in this article have these characteristics and suggests that patient-level predictions of outcome are feasible in the acute setting.

Ideally, brain tissue diffusion measurements should be independent of the MRI scanner and image acquisition parameters. However, we noted significant variations in apparent diffusion coefficient and FA values across individual scanners and at different sites. The variance was significant enough to
Table 4. Comparison of the Performance of the IMPACT and DTI Score

Estimate

$P$ Value

Training base $(\mathrm{N}=105)$ (leave-one-out scoring for the DTI model)

IMPACT AUC

DTI score AUC

$0.62(0.52-0.76)$

DTI score vs. IMPACT

$0.84(0.75-0.91)$

$\mathrm{NRI}$

DTI score vs. IMPACT IDI

$0.30(0.17-0.46)$

$<0.001$

$0.19(0.09-0.32)$

$<0.001$

Testing base $(\mathrm{N}=38)$

IMPACT AUC

$0.68(0.52-0.87)$

DTI score AUC

$0.80(0.54-0.94)$

DTI score vs. IMPACT

$0.23(-0.04$ to 0.39$)$

0.04

$\mathrm{NRI}$

DTI score vs. IMPACT

$0.16(-0.03$ to 0.36$) \quad 0.009$

Data are values $(95 \% \mathrm{Cl})$.

$\mathrm{AUC}=$ area under the receiver operating characteristic curve; $\mathrm{DTI}=$ diffusion tensor imaging; IDI = integrated discrimination improvement; IMPACT = International Mission for Prognosis and Analysis of Clinical Trials; NRI = net reclassification improvement.

seriously undermine the generalizability of our results and led us to implement a normalization step via control subjects at each center. Our normalization approach differs from the more widely used practice of scanning the same control subject(s) on all scanners, which is impractical in a multicenter study spanning a large geographical area. In addition, use of the same control subjects does not lend itself to the development of a broadly applicable outcome prediction algorithm that any center can implement using its own set of control subjects.

Some limitations of this study should be noted. First, the variables used in the IMPACT score were collected in the hyperacute phase ( $<48 \mathrm{~h}$ after injury), whereas MRI data were acquired at a later time point (an average of 3 weeks after TBI).

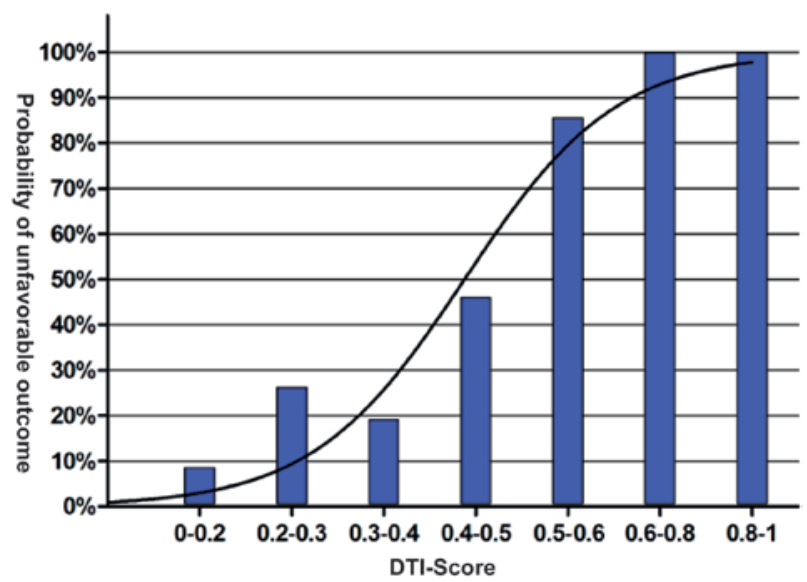

Fig. 4. Probability of having an unfavorable outcome as a function of the DTI score in the training cohort. DTI = diffusion tensor imaging. 
Favorable outcome patient (TC01048)
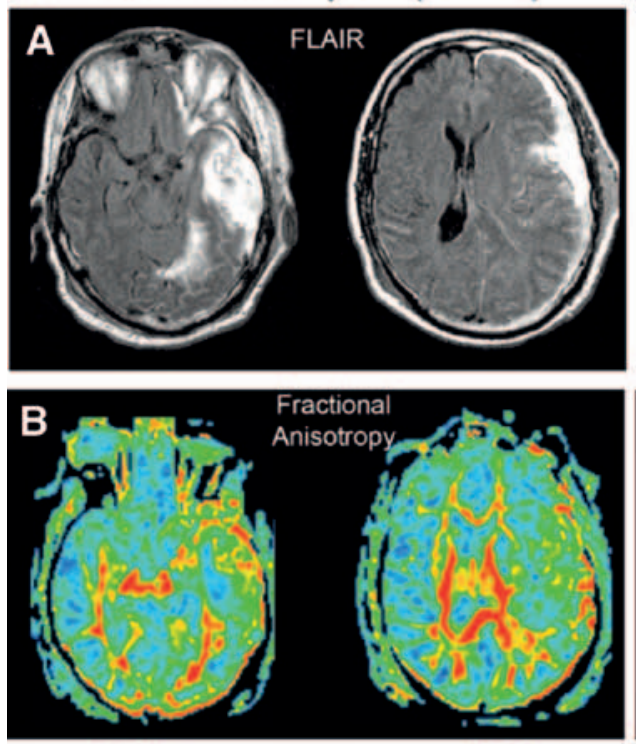

Unfavorable outcome patient (TC01015)
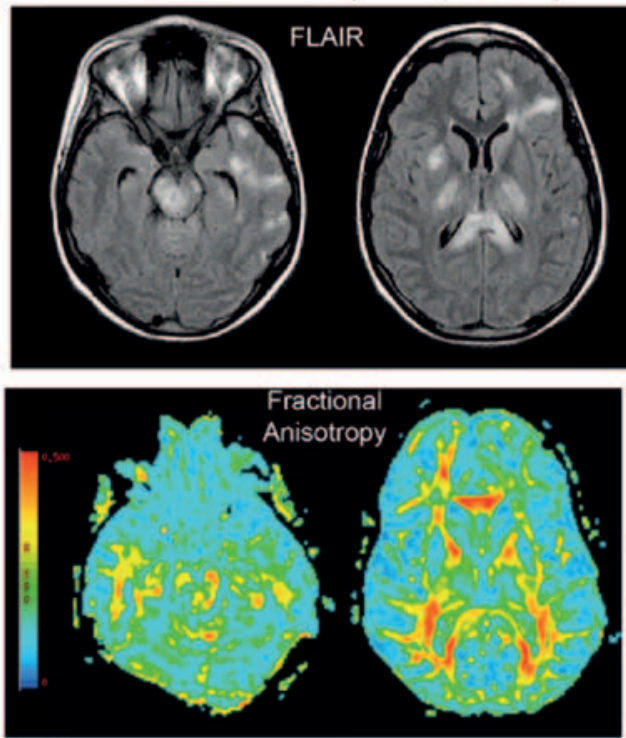
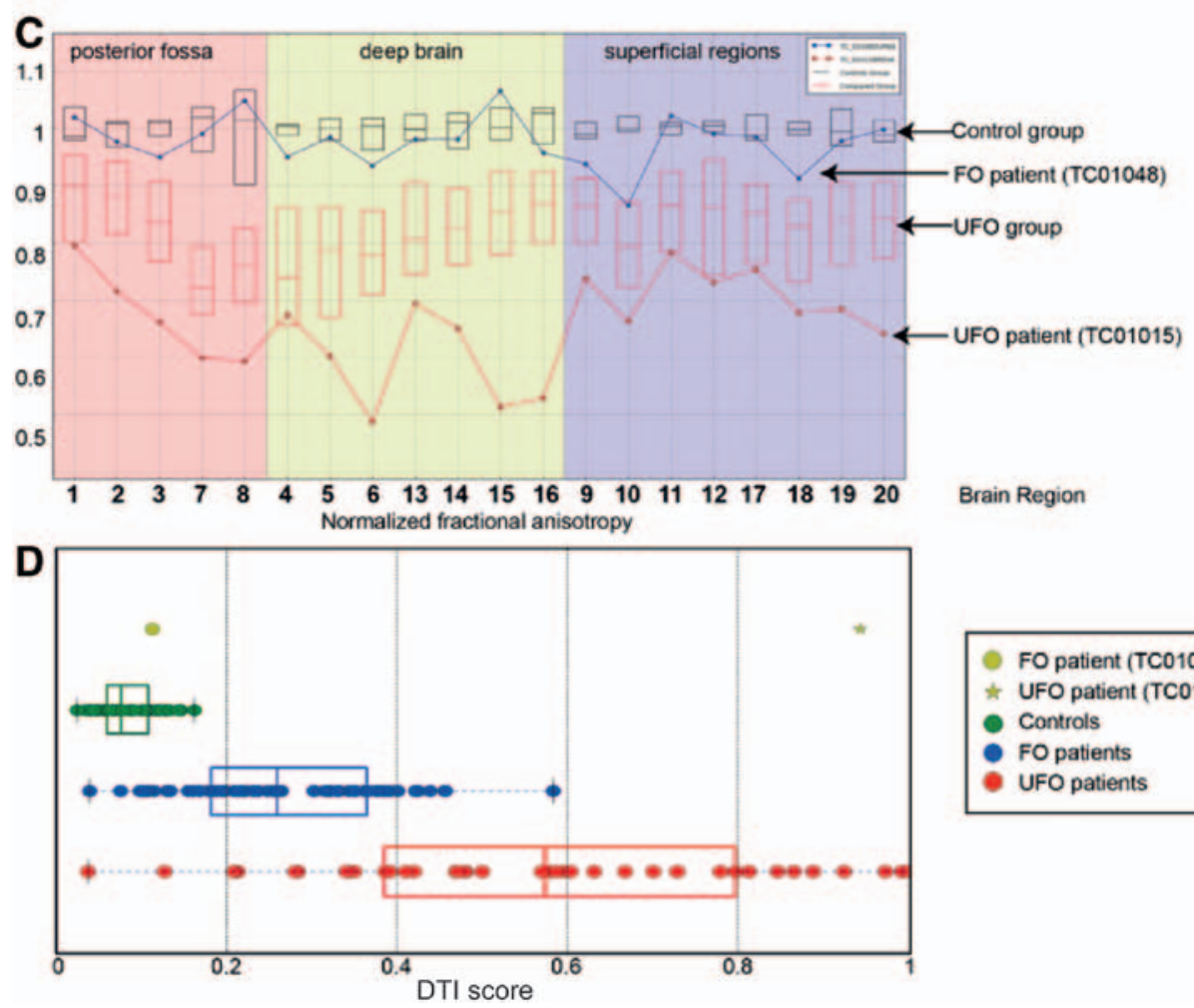

FO patient (TC01015)
* UFO patient (TC01048)
Controls
FO patients
UFO patients

Fig. 5. Conventional magnetic resonance (MR) images for two patients with FO and UFO $(A)$, fractional anisotropy (FA) maps at the same two levels $(B)$. Quantification of FA for the controls (black boxes) and patients with unfavorable outcomes (red boxes) in the 20 white matter regions considered by the study $(C)$. Each box indicates the median value and the adjacent quartiles. The FA values for these two patients are also plotted in this panel. DTI scores for these two patients, along with all the patients and all the controls, are shown in $(D)$. DTI = diffusion tensor imaging; FLAIR = Fluid Attenuated Inversion Recovery; FO = favorable outcomes; UFO = unfavorable outcomes.

Second, outcome evaluation at 1 yr was performed through a telephone interview conducted by each participating center. A central review was performed on the final data to correct for center-specific bias. Although direct clinical examination would have provided more detailed and accurate data, this was logistically prohibitive because of cost, geographical distance, and severe handicaps affecting some of the patients. Other studies have shown that remote evaluation of patients with altered consciousness provides acceptable accuracy while minimizing time and cost in data gathering. ${ }^{32}$ Third, we used a modified version of the GOS that has not been independently validated; nevertheless, we believed that this 
modification is essential to account for the fact that the "severe disability" (GOS 3) is inherently heterogeneous. Despite the difficulty of dichotomization, an issue that is intrinsic to much clinical outcome research, it is not a fundamental limitation. The methodology used here can also be used to predict the outcome probability on any given measurement scale. A scale with finer gradations and granularity (e.g., the extended GOS) will need an increased cohort size and will necessitate a larger, multicenter research consortium.

This study demonstrates that it is feasible to build a standardized, generalizable prediction system for long-term neurological outcome in critically ill comatose patients. It further establishes the feasibility of multicenter normalization to overcome data heterogeneity in quantitative DTI studies. Based on these results, one can envision a publicdomain expert system that would allow a clinician anywhere in the world to upload basic clinical information and DTI data pertaining to a particular case; the system could check the completeness and acceptability of the information and provide the probability of various outcomes on a userselected outcome scale. Other models will need to be evaluated including ones exploiting the differential association between selected white matter tracts and specific outcomes. In addition to outcome prediction, such a database could also be analyzed for other purposes including research on the genetic basis for variance in brain injury outcomes and mechanisms of postinjury repair and plasticity.

\section{References}

1. Grubb A, Walsh P, Lambe N, Murrells T, Robinson S: Survey of British clinicians' views on management of patients in persistent vegetative state. Lancet 1996; 348:35-40

2. Murray GD, Butcher I, McHugh GS, Lu J, Mushkudiani NA Maas AI, Marmarou A, Steyerberg EW: Multivariable prognostic analysis in traumatic brain injury: Results from the IMPACT study. J Neurotrauma 2007; 24:329-37

3. Lingsma HF, Roozenbeek B, Steyerberg EW, Murray GD, Maas AI: Early prognosis in traumatic brain injury: From prophecies to predictions. Lancet Neurol 2010; 9:543-54

4. Mac Donald CL, Johnson AM, Cooper D, Nelson EC, Werner NJ, Shimony JS, Snyder AZ, Raichle ME, Witherow JR, Fang R, Flaherty SF, Brody DL: Detection of blast-related traumatic brain injury in U.S. military personnel. N Engl J Med 2011 364:2091-100

5. Sidaros A, Engberg AW, Sidaros K, Liptrot MG, Herning M Petersen P, Paulson OB, Jernigan TL, Rostrup E: Diffusion tensor imaging during recovery from severe traumatic brain injury and relation to clinical outcome: A longitudinal study. Brain 2008; 131(Pt 2):559-72

6. Tollard E, Galanaud D, Perlbarg V, Sanchez-Pena P, Le Fur Y, Abdennour L, Cozzone P, Lehericy S, Chiras J, Puybasset $\mathrm{L}$ : Experience of diffusion tensor imaging and $1 \mathrm{H}$ spectroscopy for outcome prediction in severe traumatic brain injury: Preliminary results. Crit Care Med 2009; 37:1448-55

7. Pagani E, Hirsch JG, Pouwels PJ, Horsfield MA, Perego E, Gass A, Roosendaal SD, Barkhof F, Agosta F, Rovaris M, Caputo D, Giorgio A, Palace J, Marino S, De Stefano N, Ropele S, Fazekas F, Filippi M: Intercenter differences in diffusion tensor MRI acquisition. J Magn Reson Imaging 2010 31:1458-68

8. Marshall LF, Bowers MS, Klauber MR, van Berkum CM, Eisenberg HM, Jane JA, Luerssen TG, Marmarou A, Foulkes
MA: A new classification of head injury based on computerized tomography. J Neurosurg 1991; 75: S14-7

9. Smith SM, Jenkinson M, Woolrich MW, Beckmann CF, Behrens TE, Johansen-Berg H, Bannister PR, De Luca M, Drobnjak I, Flitney DE, Niazy RK, Saunders J, Vickers J, Zhang Y, De Stefano N, Brady JM, Matthews PM: Advances in functional and structural MR image analysis and implementation as FSL. Neuroimage 2004; 23(Suppl 1):S208-19

10. Pierpaoli C, Basser PJ: Toward a quantitative assessment of diffusion anisotropy. Magn Reson Med 1996; 36:893-906

11. Smith SM, Jenkinson M, Johansen-Berg H, Rueckert D, Nichols TE, Mackay CE, Watkins KE, Ciccarelli O, Cader MZ, Matthews PM, Behrens TE: Tract-based spatial statistics: Voxelwise analysis of multi-subject diffusion data. Neuroimage 2006; 31:1487-505

12. Mori S, Wakana S, Nagae-Poetscher L, Zijl PV: MRI Atlas of Human White Matter. Amsterdam, The Netherlands, Elsevier, 2005

13. Giacino JT, Ashwal S, Childs N, Cranford R, Jennett B, Katz DI, Kelly JP, Rosenberg JH, Whyte J, Zafonte RD, Zasler ND: The minimally conscious state: definition and diagnostic criteria. Neurology 2002; 58:349-53

14. Chang C-C, Lin C-J: LIBSVM: A library for support vector machines. ACM Transactions on Intelligent Systems and Technology 2011; 2:1-27

15. Hanley JA, McNeil BJ: A method of comparing the areas under receiver operating characteristic curves derived from the same cases. Radiology 1983; 148:839-43

16. Maas AI, Steyerberg EW, Butcher I, Dammers R, Lu J, Marmarou A, Mushkudiani NA, McHugh GS, Murray GD: Prognostic value of computerized tomography scan characteristics in traumatic brain injury: Results from the IMPACT study. J Neurotrauma 2007; 24:303-14

17. Steyerberg EW, Mushkudiani N, Perel P, Butcher I, Lu J, McHugh GS, Murray GD, Marmarou A, Roberts I, Habbema JD, Maas AI: Predicting outcome after traumatic brain injury: Development and international validation of prognostic scores based on admission characteristics. PLoS Med 2008; 5:e165; discussion e165

18. Mannion RJ, Cross J, Bradley P, Coles JP, Chatfield D, Carpenter A, Pickard JD, Menon DK, Hutchinson PJ: Mechanism-based MRI classification of traumatic brainstem injury and its relationship to outcome. J Neurotrauma 2007; 24:128-35

19. Yanagawa Y, Sakamoto T, Takasu A, Okada Y: Relationship between maximum intracranial pressure and traumatic lesions detected by $\mathrm{T} 2 *$-weighted imaging in diffuse axonal injury. J Trauma 2009; 66:162-5

20. Skandsen T, Kvistad KA, Solheim O, Lydersen S, Strand IH, Vik A: Prognostic value of magnetic resonance imaging in moderate and severe head injury: A prospective study of early MRI findings and one-year outcome. J Neurotrauma 2011; 28:691-9

21. Hou DJ, Tong KA, Ashwal S, Oyoyo U, Joo E, Shutter L, Obenaus A: Diffusion-weighted magnetic resonance imaging improves outcome prediction in adult traumatic brain injury. J Neurotrauma 2007; 24:1558-69

22. Ichord RN, Naim M, Pollock AN, Nance ML, Margulies SS, Christian CW: Hypoxic-ischemic injury complicates inflicted and accidental traumatic brain injury in young children: The role of diffusion-weighted imaging. J Neurotrauma 2007; 24:106-18

23. Benson RR, Meda SA, Vasudevan S, Kou Z, Govindarajan KA, Hanks RA, Millis SR, Makki M, Latif Z, Coplin W, Meythaler J, Haacke EM: Global white matter analysis of diffusion tensor images is predictive of injury severity in traumatic brain injury. J Neurotrauma 2007; 24:446-59

24. Huisman TA, Schwamm LH, Schaefer PW, Koroshetz WJ, Shetty-Alva N, Ozsunar Y, Wu O, Sorensen AG: Diffusion tensor imaging as potential biomarker of white matter injury in diffuse axonal injury. AJNR Am J Neuroradiol 2004; 25:370-6 
25. Chastain CA, Oyoyo UE, Zipperman M, Joo E, Ashwal S, Shutter LA, Tong KA: Predicting outcomes of traumatic brain injury by imaging modality and injury distribution. J Neurotrauma 2009; 26:1183-96

26. Park JH, Park SW, Kang SH, Nam TK, Min BK, Hwang SN Detection of traumatic cerebral microbleeds by susceptibilityweighted image of MRI. J Korean Neurosurg Soc 2009; 46:365-9

27. Parvizi J, Damasio AR: Neuroanatomical correlates of brainstem coma. Brain 2003; 126(Pt 7):1524-36

28. Mac Donald CL, Dikranian K, Bayly P, Holtzman D, Brody D: Diffusion tensor imaging reliably detects experimental traumatic axonal injury and indicates approximate time of injury. J Neurosci 2007; 27:11869-76

29. Mac Donald CL, Dikranian K, Song SK, Bayly PV, Holtzman DM, Brody DL: Detection of traumatic axonal injury with diffusion tensor imaging in a mouse model of traumatic brain injury. Exp Neurol 2007; 205:116-31

30. Kraus MF, Susmaras T, Caughlin BP, Walker CJ, Sweeney JA Little DM: White matter integrity and cognition in chronic traumatic brain injury: A diffusion tensor imaging study. Brain 2007; 130(Pt 10):2508-19

31. Newcombe V, Chatfield D, Outtrim J, Vowler S, Manktelow A, Cross J, Scoffings D, Coleman M, Hutchinson P, Coles J, Carpenter TA, Pickard J, Williams G, Menon D: Mapping traumatic axonal injury using diffusion tensor imaging: correlations with functional outcome. PLOS ONE 2011; 6:e19214

32. Wilson JT, Edwards P, Fiddes H, Stewart E, Teasdale GM Reliability of postal questionnaires for the Glasgow Outcome Scale. J Neurotrauma 2002; 19:999-1005

\section{Appendix: Supplementary List of Investigators}

Didier Dormont, M.D. (Professor, Department Neuroradiology, Pitié Salpêtrière Hospital, Paris, France); Lamine Abdennour, M.D. (Doctor, Neurosurgical ICU, Pitié Salpêtrière Hospital, Paris, France); Delphine Leclercq, M.D. (Doctor, Department Neuroradiology, Pitié Salpêtrière Hospital, Paris, France); Pascale
Poete, M.D. (Doctor, Neurosurgical ICU, Pitié Salpêtrière Hospital, Paris, France); Bernard Riegel, M.D. (Doctor, Neurosurgical ICU, Roger Salengro Hospital, Lille, France); Benoit Tavernier, M.D., Ph.D. (Professor, Neurosurgical ICU, Roger Salengro Hospital, Lille, France); Patrice Jissendi, M.D. (Doctor, Department of Neuroradiology, Roger Salengro Hospital, Lille, France); Christine Delmaire, M.D., Ph.D. (Doctor, Department of Neuroradiology, Roger Salengro Hospital, Lille, France); Jean-Pierre Pruvo, M.D., Ph.D. (Professor, Department of Neuroradiology, Roger Salengro Hospital, Lille, France); Philippe Gouin, M.D. (Neurosurgical ICU, Centre Hospitalier Universitaire, Rouen, France); Pierre Gildas Guitard, M.D. (Neurosurgical ICU, Centre Hospitalier Universitaire, Rouen, France); Emmanuel Gérardin, M.D., Ph.D. (Professor, Department of Neuroradiology, Centre Hospitalier Universitaire, Rouen, France); Guillaume Perot, M.D. (Doctor, Department of Neuroradiology, Centre Hospitalier Universitaire, Rouen, France); François Sztark, M.D. (Neurosurgical ICU, Centre Hospitalier Universitaire, Bordeaux, France); Vincent Dousset, M.D., Ph.D. (Professor, Department of Neuroradiology, Centre Hospitalier Universitaire, Bordeaux, France); Alain Boularan, M.D. (Neurosurgical ICU, Guy de Chauliac Hospital, Montpellier, France); Pierre François Perrigault, M.D. (Neurosurgical ICU, Guy de Chauliac Hospital, Montpellier, France); Emmanuelle Le Bars, Ph.D. (Neuroradiology, Guy de Chauliac Hospital, Montpellier, France); Alain Bonafé, M.D., Ph.D. (Neuroradiology, Guy de Chauliac Hospital, Montpellier, France); Claire Charpentier, M.D. (Neurosurgical ICU, Centre Hospitalier Universitaire, Nancy, France); Antoine Baumann, M.D. (Neurosurgical ICU, Centre Hospitalier Universitaire, Nancy, France); Claudio Di Roio, M.D. (Neurosurgical ICU, Pierre Wertheimer Hospital, Lyon, France); Dominique Sappey-Marinier, Ph.D. (Research Director, CERMEP, Lyon, France). 